\title{
Targeting Tumor Metabolism: A Biochemical Explanation Related to A Systems Biology Lipidomics Based Approach
}

\section{Vincent van Ginneken*}

Blue Green Technologies, Runderweg, Lelystad, Netherlands

\begin{abstract}
This mini-review presents the current state of our knowledge of biomarkers in the selected oncology research area of "adipose tumors": lipomas (benign) and liposarcomas. (malign). To our awareness never before have clear arguments been given underpinning a hypothesis that convincingly stated that malignant transformation from a lipoma can occur towards a liposacroma based on a biochemical model. Acidic $\mathrm{pH}$-due to lactic acid- derived from cancer cells may induce failed reprogramming of normal differentiated cells adjacent tumor cells and turn them into cancer cells. An important observation at this cellular response of aerobic fermentation ("Warburg effect") is that it occurs already at pre-carcinogenic conditions so it has another major aiming, we hypothesize to maintain the redox balance in combination with glutaminolysis and reversed $\beta$-oxidation in order to keep the Krebs cycle spinning. This peculiar observation convinced us-after studying obesity and two novel biomarkers for type 2 diabetes-that most cancers are a metabolic and redox balance disease. In addition, we describe the present role of Lipoproteins like cholesterol as carrier of anti-cancer medicine but hypothesize simultaneously that "fat particles" carried by lipoproteins can result in metastasis of "lipid tumors". We give four demands for a suitable biomarker-not only for a metabolomics based on a lipidomics based approach-but in general. The final culprit of this review is the biochemical model for this 36:1 phosphatidylcholine biomarker which was not only found in all non-adipose tissue but also in the blood. Hypoxic conditions in white adipose tissue (WAT) either during obesity, either in the microenvironment of an adipose tumor will result in further growth based of fatty acid (FA) chain elongation based on a reversal of the $\beta$-oxidation under hypoxia in order to maintain the redox balance and keep the Krebs cycle spinning.
\end{abstract}

Keywords: Lipomas; Liposacromas; Biomarkers; Oncology; Cancer; Warburg effect; Glutaminolysis; Metabolic and redox disease; Lipidomics; Phosphatidylcholine; Mitochondrial redox balance; FA chain elongation; Reversed $\beta$-oxidation

\section{Introduction}

Cancer is a group of diseases characterized by the uncontrolled growth and spread of abnormal cells and oncology is a branch of medicine that deals with tumors [1]. This review presents the current state of our knowledge of biomarkers in the selected oncology research area of lipomas (benign) and liposarcomas, (malign). A lipoma is a benign tumor composed of adipose tissue (body fat) (Figure 1). It is the most common benign form of soft tissue tumor about 1 in 100 people (1\%) will develop a lipoma [2]. Numerous case reports document the presence of lipomas in other, rare locations, with these

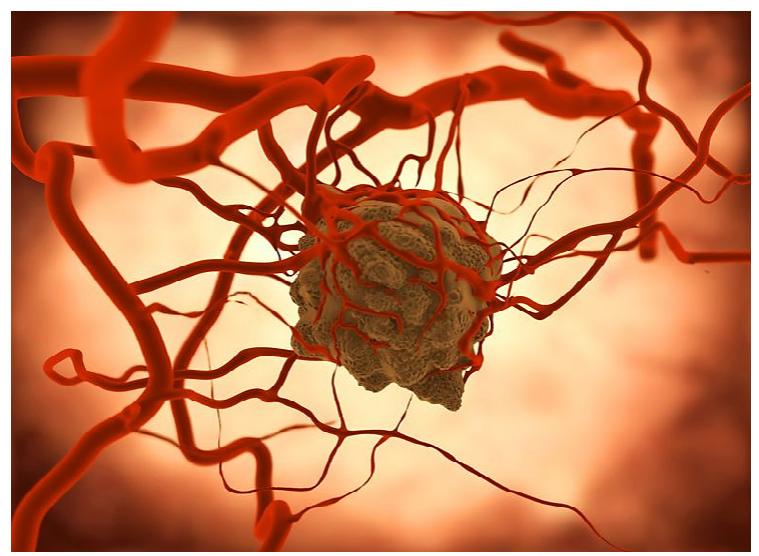

Figure 1: When an adipose tumor becomes large enough where it needs to increase the supply of nutrients and oxygen it receives, it will create new blood vessels but its microenvironment remains hypoxic [5]. tumors having been found virtually everywhere in the body. Although it has been hypothesized that lipomas may rarely undergo sarcomatous change, this event has never been convincingly documented. It is more probable that lipomas are at the benign end of the spectrum of tumors, which, at the malignant end, include liposarcomas (LS). Liposarcomas are a heterogeneous group of adipocytic tumors that represent approximately $13 \%$ of all sarcomas [3].

The WHO classification distinguishes four histologically distinct LS subtypes: atypic lipomatous tumors (ALT) (synonymously well differentiated liposarcoma, WDLS), dedifferentiated liposarcomas (DDLS), myxoid liposarcomas (MLS) and pleomorphic liposarcomas (PLS) [4].

\section{Is Transformation from a Lipoma Towards a Liposacroma an Option?}

Never before have clear arguments been given underpinning a hypothesis that convincingly stated that malignant transformation from a lipoma can occur towards a liposacroma based on a biochemical model. An increased rate of lipid synthesis in cancerous tissues has long been recognized as an important aspect of the rewired metabolism of transformed cells. However, the contribution of lipids to cellular

*Corresponding author: Vincent van Ginneken, Blue Green Technologies, Runderweg 6, 8219 Lelystad, Netherlands, Tel: 0031638071180; E-mail: vvanginneken@hotmail.com

Received November 15, 2016; Accepted January 26, 2017; Published January 28, 2017

Citation: Ginneken V (2017) Targeting Tumor Metabolism: A Biochemical Explanation Related to A Systems Biology Lipidomics Based Approach. J Mol Biomark Diagn S2: 022. doi:10.4172/2155-9929.S2-022

Copyright: @ 2017 Ginneken V. This is an open-access article distributed under the terms of the Creative Commons Attribution License, which permits unrestricted use, distribution, and reproduction in any medium, provided the original author and source are credited 
transformation, tumor development and tumor progression as well as their potential role in facilitating the spread of cancerous cells to secondary sites, are not yet fully understood [5,6]. In this review, we will attempt to give an explaining biochemical model under section [5].

Speculation exists regarding a potential link between traumainduced cytokine release triggers pre-adipocyte differentiation and maturation. To date, no definitive link between trauma and lipoma formation has been prospectively demonstrated [7].

Also, an association with gene rearrangements of chromosome 12 has been established in cases of solitary lipomas, as has an abnormality in the HMGA2-LPP fusion gene [8].

I will review the similarities between obesity and type 2 diabetes as a consequence of a high fat diet and these forms of "adipose" oncogenic cancers. Hypoxia as a pathological hallmark feature of solid tumors as well as WAT. Characteristic for common fat tissue (white adipose tissue WAT) is that it has a low vascularization degree so that low oxygen conditions (hypoxia) can occur (Figure 2).

Though hypoxia is an adverse physiological state, tumors have evolved to utilize this unsuitable environment to their own advantage by activating key biochemical and cellular pathways that are important in progression, survival, and metastasis. Several studies have emphasized the importance of lipid mediators in regulating key biomolecules in the hypoxic microenvironment, for example hypoxia inducible factor-1 (HIF-1), the master regulator of hypoxia. Lipid mediators have been reported to enhance the levels and activity of HIF-1, which subsequently signal to stimulate angiogenesis and tumor cell survival under hypoxic conditions. There are also reports of hypoxia and HIF-1 enhancing the levels of some lipid mediators mostly by upregulating the levels of the enzymes responsible for their biosynthesis [5].

This review gives a brief outline of the biochemical mechanism and the role played by bioactive lipid mediators in the regulation of tumor progression and survival under conditions of hypoxia. Not solely around the belly but also due to "the lipid overflow theory" [9], resulting in a metastasis through the fat particles of e.g. Cholesterol (Ch) or Triacylglycerols (TGs) circulating through the body attached to lipoproteins.

The question remains how a benign hypoxic "lipid tumor" can become malign. As a tumor grows, it rapidly outgrows its blood supply, leaving portions of the tumor with regions where the oxygen concentration is significantly lower than in healthy tissues. Hypoxic

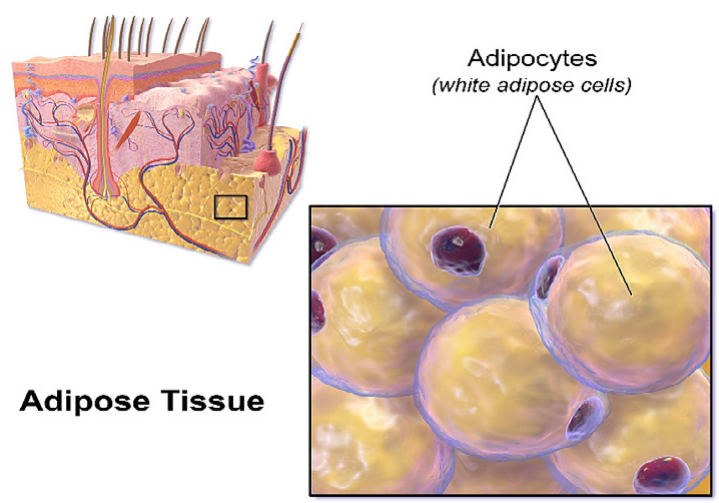

Figure 2: White Adipose tissue has a low vascularization so that low oxygen conditions (hypoxia) can occur. micro-environments in solid tumors are a result of available oxygen being consumed within $70 \mu \mathrm{m}$ to $150 \mu \mathrm{m}$ of tumor vasculature by rapidly proliferating tumor cells thus limiting the amount of oxygen available to diffuse further into the tumor tissue. In order to support continuous growth and proliferation in challenging hypoxic environments, cancer cells are found to alter their metabolism. It is thought that the low $\mathrm{pH}$ of hypoxic tumors in result of high levels of lactic acid can promote tumor cell invasion by destruction of adjacent non-cancerous tissue [10-12].

Carbonic anhydrase involved in maintaining a slightly alkaline intracellular $\mathrm{pH}$ does so by removing carbonate from the extracellular space consequently acidifying the cells surroundings. Moreover, proton pumping from the hypoxic tumor cells further decreases the surrounding $\mathrm{pH}$. With the hypoxic tumor cells consuming large amounts of glucose to maintain energy homeostasis, the tumor has found a way of using its resources most efficiently. The end glycolytic product of hypoxic tumors, lactate, is transported out of the hypoxic cell by monocarboxylate transporter 4 (MCT4) which is a hypoxia induced transporter. The free lactate in the extracellular space is then taken up by monocarboxylate transporter 1 (MCT1) which is a nonhypoxia induced transporter found on the surface of aerobic cells. This transporter allows aerobic cancer cells to efficiently take up lactate, convert it back to pyruvate with the oxygen-dependent expression of lactate dehydrogenase B (LDH-B), and use it as an energy source. This frees these cells from requiring large quantities of glucose allowing the hypoxic cells to take up the majority of the available resources [12].

Acidic $\mathrm{pH}$ derived from cancer cells may induce failed reprogramming of normal differentiated cells adjacent tumor cells and turn them into cancer cells. Genetic and epigenetic modification in cells via acidic $\mathrm{pH}$. This hypothesis posted by [13] deals with the ability of acidic $\mathrm{pH}$ to convert normal cells adjacent cancer cells to cancerous cells and its inductive potential on genetic and epigenetic modification of normal cells adjacent cancer cells and in this hypothesis lactic acid release is a tool to up-regulate cell reprogramming markers, induce epigenetic modification and tumor progress in normal cells adjacent cancer cells [13].

\section{Cancer as Metabolic and Redox Disease}

Over the past century, traditionally medical care has centered on standards of care based on epidemiological studies of large cohorts. However, large cohort studies do not take into account the genetic variability of individuals within a population e.g. at the level of genomics, according the national human genome research (NHGRI) about $99.9 \%$ of the DNA sequence is identical in all people, but the $0.1 \%$ difference is critical because it represents the genetic variations in the susceptibility of a person for getting a disease, the severity of the disease, and what is the impact of the medical/therapeutic treatment [14]. Moreover, it is the general belief cancer is caused by mutations and altered gene expression, in a process called malignant transformation, resulting in an uncontrolled growth of cells [15]. In this respect expression of genes responsible for glycolytic enzymes and glucose transporters are enhanced by numerous oncogenes including RAS, SRC, and MYC and cellular mediators of signal transduction and gene expression, including the phosphatidylinositol 3-kinase (PI3K)/Akt/mTOR system, HIF-1 and Myc, during physiologic cell proliferation and tumorigenesis [16].

In our opinion the advent of molecular cancer genetics diverted biologists away from studies of cancer cell metabolism. Our thinking, that some cancers are a metabolic disease came from the literature 


\begin{tabular}{|c|c|c|c|}
\hline Change in hepatic expression & 40-days Control-diet & 40-days High-Fat diet & P-value \\
\hline$\Delta$ 6-desaturase & $0.031 \pm 0.001$ & $0.092 \pm 0.006$ & \% change \\
\hline Elongase & $1.046 \pm 0.092$ & $3.181 \pm 0.617$ & $296.3 \% \uparrow$ \\
\hline$\Delta$-desaturase & $12.751 \pm 0.629$ & $3.911 \pm 0.768$ & $\mathrm{P} \leq 0.0003^{* * *}$ \\
\hline$\Delta$-desaturase & $2.661 \pm 0.532$ & $8.911 \pm 1.200$ & $\mathrm{P} \leq 0.00000^{\star * * *}$ \\
\hline
\end{tabular}

Table 1: Enzyme activity of desaturases and elongases in blood plasma. With LC-MS techniques the individual fatty acids of the Cholesterol fraction in blood plasma were determined in a 40 days Control-diet $(\mathrm{N}=5)$ and a 40 days high-fat diet $(\mathrm{N}=6), \mathrm{C} 57 \mathrm{BI} / 6$ mouse group. Endogenous conversions by desaturases and elongase were estimated from product-to-precursor ratios as given in the research work of [29].
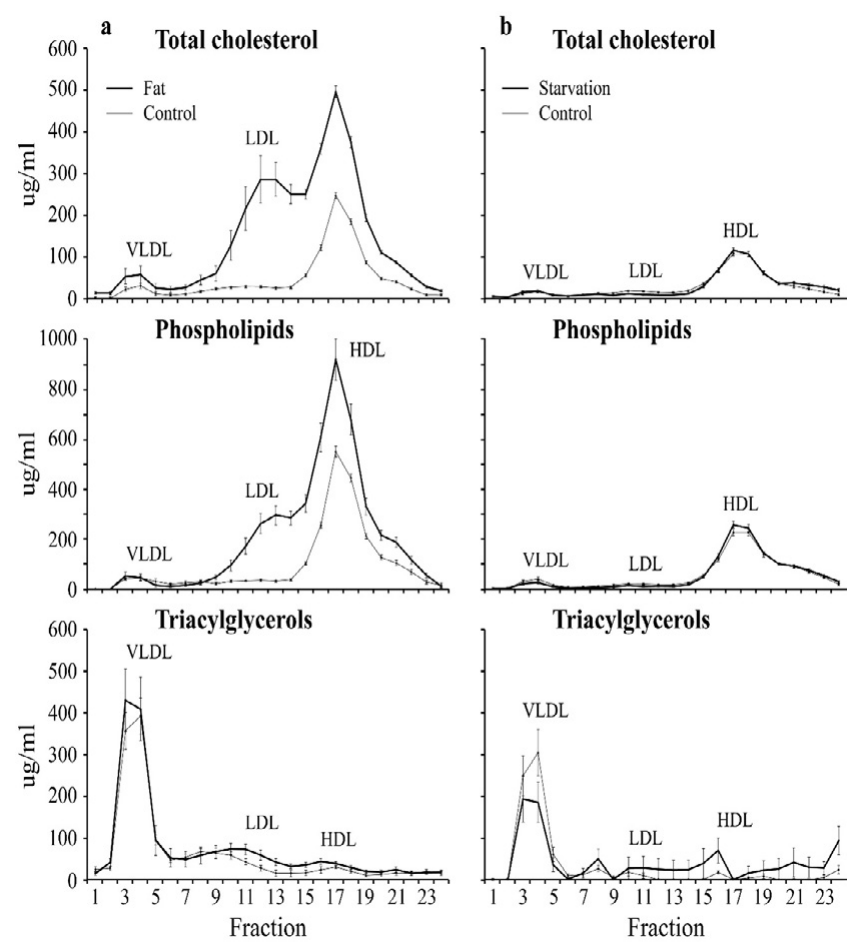

Triacylglycerols

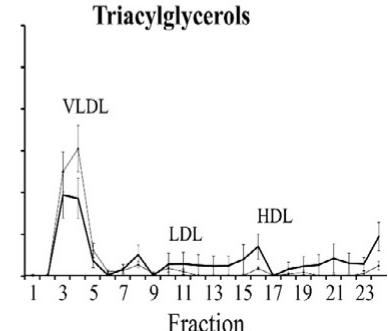

Figure 3: Lipoprotein distribution-profiles. Effect of starvation and a High-Fa diet on cholesterol-, phospholipids- and triacylglycerols levels in plasma and the effect on the distribution of those lipids over different lipoproteins in plasma. Fractions 3-7 represent VLDL, fractions 8-15 LDL and fractions 15-19 represent HDL (Mean \pm SD). Number of animals Control A $(n=6)$, Treatment $A(n=6)$, Control B $(n=6)$, Treatment B $(n=7)$. Source: [10]

describing the "Warburg effect" (see further) which is of the high flux/ low efficiency type. An important observation at this cellular response of aerobic fermentation is that it occurs already at pre-carcinogenic conditions so it has another major aiming. This peculiar observation convinced us -after studying obesity and two novel biomarkers for type 2 diabetes [17]-that most cancers are a metabolic and redox balance disease. So, we hypothesize that some oncology cancers with an "fat composed tumor" might be related to a similar mechanism and that the disturbed hypoxic cell solely has the aiming to produce $\mathrm{NAD}(+)$ in order to keep the cellular redox potential maintained and the Krebs cycle spinning. In other studies, several oncogenes and tumor suppressors have been linked to the regulation of metabolic processes $[18,19]$, which give supportive evidence to follow this research line.

\section{Cancer Can be Induced by Obesity}

For decades, it was already known that unhealthy food, a sedentary lifestyle and smoking were possible causes for cancer [20]. A very recent study by the International agency for research on cancer handbook working group (IARC) workgroup in the NEJM study proved-based on more than 1000 epidemiologic studies which were observational studies on cancer risk and excess body fatness-that obesity is directly correlated to several types of cancer [21].

More specific, the IARC workgroup identified an additional eight cancers for which there is now also sufficient evidence that the absence of body fatness lowers cancer risk, including cancers of the gastric cardia, liver, gallbladder, pancreas, ovary, and thyroid, as well as multiple myeloma and meningioma. (For detailed information on the evaluation criteria, see the working procedures section of the IARC Handbooks of Cancer Prevention website at http://handbooks. iarc.fr/workingprocedures/index1.php). For cancers of the colon, rectum, gastric cardia, liver, gallbladder, pancreas, and kidney and for esophageal adenocarcinoma, significant associations between BMI and cancer risk were reported, with positive dose-response relationships [21]. So, by this extended study in NEJM a clear correlation between Body Fatness and cancer has been proven.

\section{Hypoxia Results in Further Fat Synthesis and Tumor Growth}

In order to conserve the energy from glucose as ATP, three major metabolic pathways are involved three metabolic pathways are involved in the mitochondria (Figure 3), directly linked to the oxidative phosphorylation outside in the cytosol.

The glycolysis, the Krebs-cycle (TAC) and the $\beta$-oxidation [22,23]. So, our perception is that in white adipose tissue (WAT) low oxygen (hypoxia) are the case as outlined in the research article of [24] and the review of [25]. We stated earlier and depicted in Figure 1 that in WAT ischemic and/or hypoxic conditions may occur. Under these conditions, it can be questioned how the Krebs cycle persist in its activity and the redox balance maintained. Since the early work at hypoxic conditions at fish clearly reviewed and outlined by [6] and recently in tumor research [26,27] two hypothesis has been postulated related to these two research topics: fatty chain elongation and anaerobic endogenous reduction of unsaturated fatty acids [21,23].

We observed in our submitted "biomarker publication" [17] based on product-to-precursor ratio ratios [28] significant high $\left(\mathrm{P}<0.0003^{* * *}\right)$ elongase activity (Table 1) under High-Fat diet conditions significantly which increased with $304 \%$. So, high-fat diet conditions lead to fat formation ( $\approx$ white adopise tissue (WAT)) according to elongase/ desaturase enzymatic acitivity (Table 1).

In the "overall" publication [17] of an obese/type-2 diabetes C57bl6 mouse model these fatty chain elongation data are given an explanation mentioned following below mentioned described mechanism of reversed $\beta$-oxidation to maintain the redox balance and keep the Krebs cycle spinning resulting in anaerobic fatty chain elongation and consequently fat (WAT) formation [17].

The concept of anaerobic fatty chain elongation involves the coupling of acetyl-CoA units to fatty acids via a reversal of the $\beta$-oxidation. The first three reactions are identical to the $\beta$-oxidation. However, the last reaction is catalyzed by the NAD-dependent 
enoyl-CoA reductase instead of the Co-Q dependent acetyl-Co-A dehydrogenase. In this way, the reaction is thermodynamically favored in the direction of chain elongation which corresponds to fat synthesis

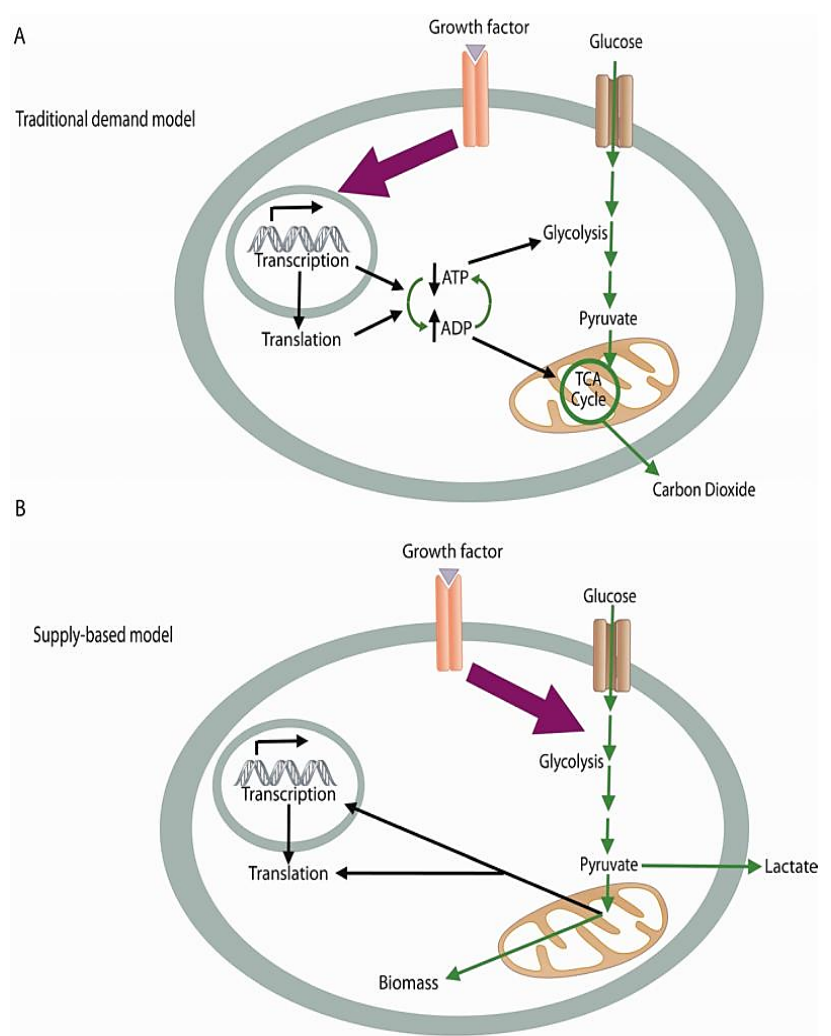

Figure 4: Until presently it is believed "derailed" cancer cells are the consequence of an environmental growth factor in combination with genetic factors the so called "onco-genes". The combination of both results in the production of tremendous amount of biomass-the growth of the tumor- based on every run the Krebs cycle is spinning. In contrast, we hypothesize most cancers are a metabolic and redox-balance disease (Section 5 and 6).

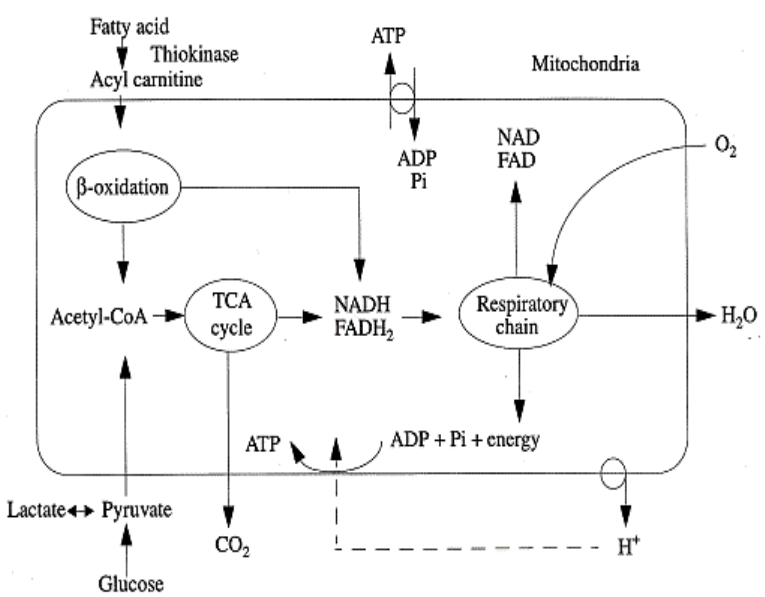

Figure 5: Biochemical overview of the three major pathways in the mitochondria: the glycolysis, the Krebs cycle and the $\beta$-oxidation. Under hypoxic conditions-which is the case in excessive WAT-tissue- both the Krebscycle and the $\beta$-oxidation needs to maintain their redox-balance which is on one hand performed lipid synthesis and by "reversed $\beta$ oxidation" (fatty chain elongation) also leading to fat synthesis $=>$ so a vicious circle is observed during severe obesity $[22,23]$.
[29]. Since fatty chain elongation consumes 2 moles of NADH in each cycle, this pathway provides a suitable mechanism to maintain the mitochondrial redox balance [22,23] (Figure 4).

Indeed, fatty acid chain elongation (i.e. lipid synthesis) during anoxia could be stimulated in vitro by addition of Krebs-cycle intermediates such as glutamine [27] which is indicative for the redox coupling between Krebs-cycle activity and fatty acid chain elongation or "fat formation" (WAT) [29-31].

Fatty acid chain elongation (i.e. lipid synthesis) during anoxia/ ischemia has been demonstrated in various organisms starting with invertebrate models and finding its way towards biomedicine [32]. But most important for cardiovascular diseases FA chain elongation has been observed in ischemic arterial tissue [33] and ischemic mammalian heart [34]. In the sections above, the lipid metabolism has been proposed as a suitable mechanism to maintain redox balance in anoxic tolerant invertebrates [32]. However, hypoxia may have also adverse effects on lipid metabolism, resulting in a decreased cellular functional integrity and eventually tissue damage. Especially in mammalian tissues being essentially hypoxia intolerant, these effects have received large attention [35].

When oxygen availabilityis decreased, theoxidative phosphorylation is inhibited and as a result of the accumulating reducing equivalents $\left(\mathrm{NADH}, \mathrm{FADH}_{2}\right)$, the fluxes through the Krebs-cycle and $\beta$-oxidation are impaired. Since the flux through the $\beta$-oxidation is high during normoxic conditions [22,23], fatty acids and their metabolites rapidly accumulate during anaerobiosis [34] (Figure 5).

In addition, during hypoxia or ischemia, the cellular energy status may be affected. A number of ionic rearrangements occur due to the inhibition of ATP-dependent membrane associated ion pumps and intracellular acidosis. This may activate phospholipases which results in a marked hydrolysis of membrane lipids $[22,23,28,35]$.

Fatty acid synthesis is a paradigm of tumor biosynthetic pathways because it requires the use of a Krebs cycle intermediate (citrate) that might otherwise be oxidized in the mitochondria. The shunting of metabolites from the Krebs cycle into other pathways (cataplerosis) is part of the fundamental biochemistry of cell growth, and it emphasizes the versatility of the Krebs cycle: rather than serving a purely oxidative function as a source of reducing equivalents for the electron transport chain (ETC), it can also be used as a continuous source of precursor molecules for biosynthetic pathways. Early characterization of cataplerosis in highly lipogenic hepatoma cells led to the concept of a "broken" or "truncated" Krebs cycle because of an apparent impairment in citrate oxidation and the demonstration that the rate of citrate export was directly proportional to the rate of cell proliferation [36].

In an earlier manuscript, we demonstrated a "solution" in an obese C57 mouse model of reversed $\beta$-oxidation in order to maintain the redox balance and keep the Krebs cycle spinning [17]. This modelincluding fatty chain elongation-ultimately leads to fat (WAT) formation.

\section{Some Cancers are a Metabolic and Redox Disease}

Primary features the type of cancer we describe -"a fat composed tumor"- are maintained via intrinsically modified metabolic activity, which is characterized by enhanced nutrient supply, energy production, and biosynthetic activity to synthesize a variety of macromolecular components during each passage through the cell cycle. This metabolic shift in transformed cells, as compared with nonproliferating cells, involves aberrant activation of aerobic glycolysis, 
de novo lipid biosynthesis and glutamine-dependent anaplerosis to fuel robust cell growth and proliferation. Here, we discuss the unique metabolic characteristics of cancer, the constitutive regulation of metabolism through a variety of signal transduction pathways and/ or enzymes involved in metabolic reprogramming in cancer cells, and their implications in cancer diagnosis and therapy. Our model is that with every spinning of the Krebs cycle cell proliferation occurs with requires nutrients, energy, and biosynthetic activity to duplicate all macromolecular components during each passage through the cell cycle. It is therefore not surprising that metabolic activities in proliferating cells are fundamentally different from those in nonproliferating cells. So, in order to keep the Krebs cycle spinning the redox balance needs to be maintained. This review examines the idea that several core fluxes form a stereotyped platform supporting proliferation of diverse cell types for:

i. aerobic glycolysis (Warburg effect);

\section{ii. de novo lipid biosynthesis;}

iii. glutamine-dependent anaplerosis,

Ad i). A particular change in metabolism, historically known as the Warburg effect [37-39] results in high rates of glycolysis in both normoxic and hypoxic cancer cells. The current popular opinion is that cancer cells ferment glucose while keeping up the same level of respiration that was present before the process of carcinogenesis, and thus the Warburg effect would be defined as the observation that cancer cells compared to normal cells, strongly upregulate glucose uptake and glycolysis to give rise to increased yield of intermediate glycolytic metabolites and the end product pyruvate. Moreover, glycolysis is uncoupled from the mitochondrial tricarboxylic acid (Krebs) cycle and oxidative phosphorylation (OXPHOS) in cancer cells. Consequently, the majority of glycolysis-derived pyruvate is diverted to lactate fermentation and kept away from mitochondrial oxidative metabolism.

Ad ii). Anaplerotic reactions are chemical reactions that form intermediates of a metabolic pathway. Examples of such are found in the citric acid cycle (Krebs cycle). In normal function of this cycle for respiration, concentrations of Krebs intermediates remain constant; however, many biosynthetic reactions also use these molecules as a substrate. Anaplerosis is the act of replenishing Krebs cycle intermediates that have been extracted for biosynthesis (in what are called cataplerotic reactions).The Krebs cycle is a hub of metabolism,

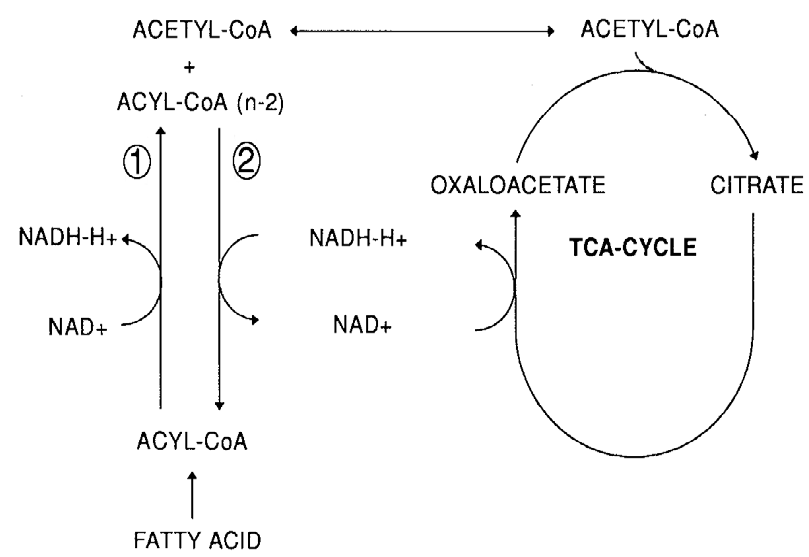

Figure 6: Schematic representation of the redox coupling between the KREBScycle and fatty acid chain elongation. (1): Normal $\beta$-oxidation; (2): reversed $\beta$-oxidation [26].

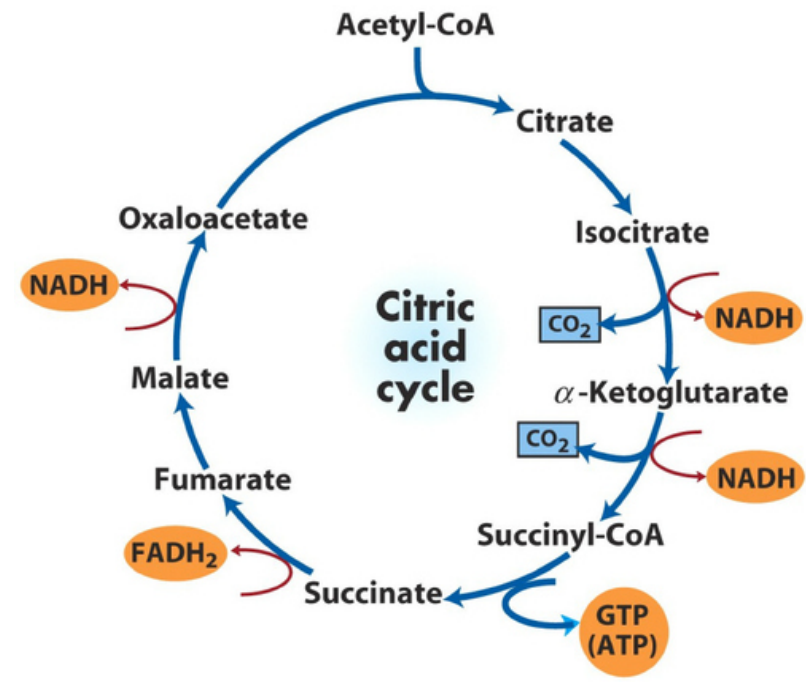

Figure 7: Spinning Krebs cycle.

with central importance in both energy production and biosynthesis. Therefore, it is crucial for the cell to regulate concentrations of Krebs cycle metabolites in the mitochondria and keep the Krebs cycle spinning. Anaplerotic flux must balance cataplerotic flux in order to retain homeostasis of cellular metabolism [39].

Ad iii). Glutamine is the most abundant amino acid in human plasma and participates in many metabolic pathways required for normal cell function. In addition to its role in protein synthesis, it provides nitrogen for the synthesis of nonessential amino acids, purines, pyrimidines, and hexosamines, and is the major source of glutamate used for glutathione synthesis [22,23] (Figure 6).

Some cancer cell lines also display addiction to glutamine despite the fact that glutamine is a nonessential amino acid that can be synthesized from glucose. The high rate of glutamine uptake exhibited by glutamine-dependent cells does not appear to result solely from its role as a nitrogen donor in nucleotide and amino acid biosynthesis. Instead, glutamine plays a required role in the uptake of essential amino acid and in maintaining activation of TOR kinase. Moreover, in many cancer cells, glutamine is the primary mitochondrial substrate and is required to maintain mitochondrial membrane potential and integrity as well as support of the NADPH production needed for redox control and macromolecular synthesis. Studies with several cancer cell lines highlight a major role for glutamine in maintaining redox balance in cancer cells that lack glucose-dependent anaplerosis [15].

Some tumor cell lines have long been known to consume glutamine at high rates in vivo and to require high concentrations of glutamine to survive and proliferate in vitro. Classical studies on tumor cell metabolism in culture demonstrated that glutamine is an important carbon source since most of the glutamine consumed is used as a respiratory substrate in the mitochondria rather than for protein synthesis. Glutamine can also be used to produce oxaloacetate during anaplerotic reactions in various cell types through "glutaminolysis", which is also seen in many c-Myc transformed cell [40] (Figure 7).

"Glutaminolysis" provides metabolic intermediates such as alphaketoglutarate to feed and thereby maintain the tricarboxylic acid cycle as a biosynthetic hub. Tumor cells fuel their metabolism with glucose and glutamine to meet the bioenergetic and biosynthetic demands of proliferation. Hypoxia and oncogenic mutations drive 

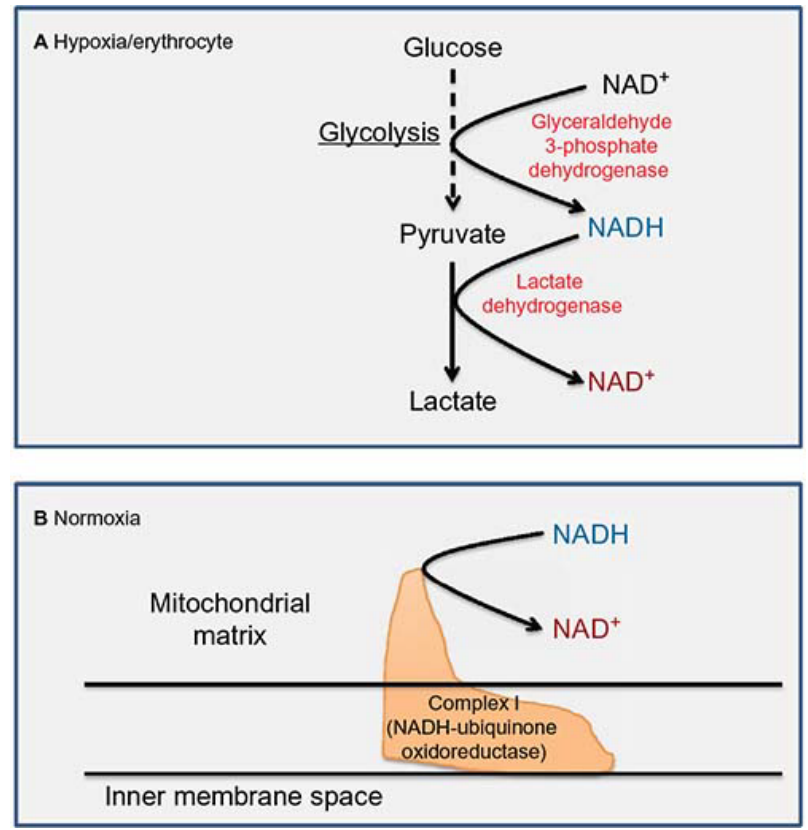

Figure 8: We postulate the hypothesis that the pre-carcinogenic Warburg effect solely serves to produce NAD+ in order to maintain the cellular redox balance. As a consequence of tremendous lactic cytosolic acid production DNA making the tumor cell malign. Further growth of the tumor cell occurs due to reversed $\beta$-oxidation in order to maintain the redox balance and keep the Krebs cycle spinning. This model-including fatty chain elongationultimately leads to fat (WAT) formation. glutamine-dependent anaplerosis in some cancer cell lines also serves to produce NAD+. So, we hypothesize that most adipose cancers-induced by a High Fat diet and obesity [21]- are a metabolic and redox balance disease.

glycolysis, with the pyruvate to lactate conversion being promoted by increased expression of lactate dehydrogenase A and inactivation of pyruvate dehydrogenase. The NAD+ pool is consecutively regenerated and supports the high glycolytic flux required to produce anabolic intermediates [41]. Glycolysis and glutaminolysis share the capacity to generate NADPH, from the pentose phosphate pathway and through the malate conversion into pyruvate, respectively. The pentose phosphate pathway is a metabolic pathway parallel to glycolysis. It generates NADPH and pentoses (5-carbon sugars) as well as Ribose 5-phosphate, the last one a precursor for the synthesis of nucleotides $[22,23]$.

\section{The Present role of Lipoproteins like Cholesterol as Carrier of Anti-Cancer Medicine}

Because blood is a transport medium that carries nutrients, lipids and waste products between cells we hypothesize the lipoproteins might play a role in in cancer cell transport. The liver takes up free fatty acids (FFA) from plasma, which are transported by lipoproteins as triacylglycerols (TG). Lipoproteins are water-soluble protein complexes, which consist of a hydrophobic core, containing triacylglycerols (TG) and cholesterol esters (CE), and a hydrophilic monolayered shell, composed of phospholipids (PL), free cholesterol (FC), and specific proteins (apolipoproteins) [42].

Five major classes of lipoproteins can be distinguished in blood plasma, including chylomicrons, very-low-density lipoproteins (VLDL), intermediate-density lipoproteins (IDL), low-density lipoproteins (LDL) and high density lipoproteins (HDL). The function of LDL and VLDL is to transport CE from and to the liver and other organs, while HDL removes cholesterol (Ch) from peripheral tissues [43].

Because dividing cancer cells needs large amounts of cholesterol to make new cellular membranes we highlight the importance of lowdensity lipoprotein (LDL) and the application of the development of bionanoparticles (BNPs) specifically related to transport cholesterol to cells expressing the LDL receptors (LDLRs). Anti-cancer drugs can be loaded onto HDL or LDL bionanoparticles (BNPs) by surface loading, core loading, and apoprotein interaction. As a result, lipoprotein nanoplatforms may provide a solution to the biocompatibility issues associated with most synthetic nanostructures via low density lipoprotein (LDL) or high density lipoproteins (HDL) like cholesterol [44].

Lipoproteins like Cholesterol and other fatty acids (FA) are not recognized as foreign entities by the human immune system and are not absorbed by the reticuloendothelial system (RES) and therefore they are they are suitable as endogenous carriers. Recently, conjugation of cholesterol moiety to active compounds for either cancer treatment or diagnosis has been considered an attractive approach either in vitro or in vivo using animal models to enhance their pharmacokinetic behavior, cellular uptake, target specificity, and safety. Research was focused on the low density lipoprotein (HDL) cholesterol and subsequent dissemination of tumor cells and formation of metastases, which constitute the main cause of cancer mortality $[45,46]$ (Figure 8).

\section{Can a Novel Type-2 Diabetes Phosphatidylcholine be Used as a Biomarker in the Hypoxic Tumor?}

Landmark discoveries in biology and medicine are sometimes a matter of serendipity than of conscious decision. Recently, we performed a study which was designed to find via a high-fat (HF) diet induced insulin resistant (IR) and/or type-2 diabetes (T2DM) C57Bl/6 mouse model potential novel biomarkers. Major aiming was to find following this lipidomics based approach novel safe biomarkers applicable for humans with IR/T2DM that can be used in the assessment of diagnosis, intensive treatment, clinical use and new drug development. At experimental animal (mouse) level a prerequisite for a biomarker is that it can be distinguished from the Control group fed a ControlChow e.g. in comparison to our, for 40 days fed a High Fat diet for our C57BL/6 mouse model resulting in obese IR/T2DM animals e. g. by an elevated concentration in an organ or tissue:

a) For biomarkers with a Metabolomics background in terms of product-precursor ratio the metabolic route is obstructed, or the enzyme cannot convert in its metabolic route the precursor to its end product resulting in an accumulation of the precursor. This means that the metabolic pathways or enzymes are blocked in this way resulting in an accumulation of the end product: the biomarker $[28,46]$.

b) A second prerequisite is that it may not be diet induced which means that it may not be found in the control-chow or high-fat diet.

c) A third requisite for a biomarker in the field is that organ/ tissue sampling by biopsy is very uncomfortable, not practical, and sometimes dangerous for e.g. a liver biopsy [46] or impossible in case of heart muscle [47]. Therefore, the third precondition for a suitable biomarker is that it not only accumulates in the specific organ or tissue but also in the blood-plasma $[17,47,48]$. 
d) In addition, the biomarker has to be found in blood-plasma simultaneously while is not a component of the HF-diet. Until recently regulatory functions of miRNAs in liposarcoma progression were investigated as potential diagnostic biomarkers in liposarcoma with a main focus on circulating miRNAs for fast and reliable differential diagnosis [49].

To our awareness never before biomarkers from lipidomics were applied in adipose tumor research. Our observations indicated a 36:1 phosphatidylcholine (36:1-PC) biomarker, which rose significantly $(\mathrm{P} \leq 0.025)$ mainly in heart muscle with $400 \%$ while concentrations increased significantly strongly $(\mathrm{P} \leq 0.002)$ in blood plasma with $1493 \%$ in the high-fat diet vs. Co. As an earlier defined prerequisite, this compound was not found in the food $[15,17]$. We hypothesized that in excessive white adipose tissue (WAT) hypoxic conditions might occur with evolving biochemical consequences [17] (Figure 9).

\section{Examples of Oncological Research Related to Phosphatidylcholine Biomarkers}

Though hypoxia is an adverse physiological state, tumors have evolved to utilize this unsuitable environment to their own advantage by activating key biochemical and cellular pathways that are important in progression, survival, and metastasis. Several studies have emphasized the importance of lipid mediators in regulating key biomolecules in the hypoxic microenvironment, for example hypoxia inducible factor-1 (HIF-1), the master regulator of hypoxia [48]. Altered phosphatidylcholine (PC) metabolism in epithelial ovarian cancer (EOC) can provide choline-based imaging approaches as powerful tools to improve diagnosis and identify new therapeutic targets $[48,50]$.

In general, in a recent article in the Lancet a multicenter, observational study evaluated the utility of molecular and genetic analysis in improving diagnostic accuracy in 384 sarcoma patients. Diagnosis was modified in $53(13.8 \%)$ cases. Of these, primary management and prognosis was altered in 45 cases, most commonly in dedifferentiated liposarcoma (23\%), dermatofibrosarcoma (16\%), and synovial sarcoma (16\%) [51]. It was concluded that molecular genetic testing should be added to histological assessment to improve diagnostic accuracy in sarcoma cancer research [50].

\section{Liposome for Drug Delivery}

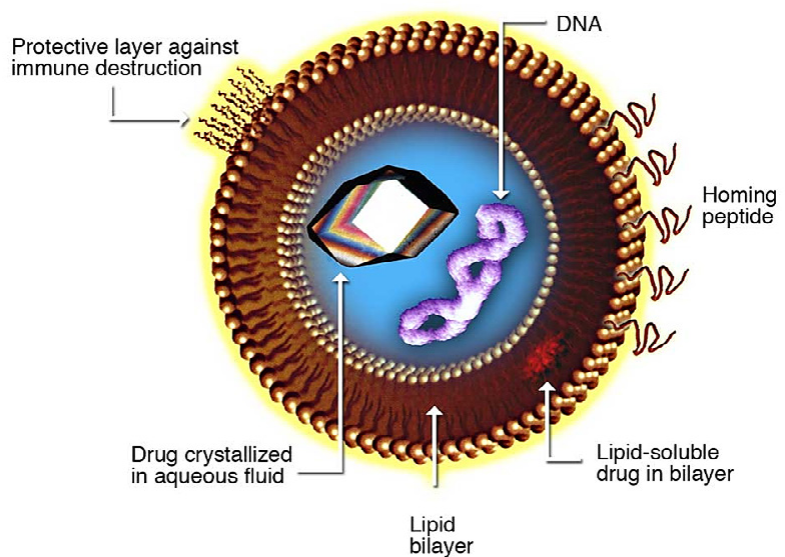

Figure 9: The use of nanotechnology in cancer treatment offers some exciting possibilities, including the possibility of destroying cancer tumors with minimal damage to healthy tissue and organs, as well as the detection and elimination of cancer cells before they form tumors $[45,46]$.
This observation of the role lysophospholipids in early stage EOC as biomarker was confirmed with satisfactory diagnostic accuracy in a clinical study of [51]. In addition -among else the phosphatidylcholine biomarkers- were specifically validated in the EOC nude mouse model giving as suggested the significant potential for diagnosing early ovarian cancer and investigating its underlying mechanisms [51]. Solely this form of cancer can in our described approach with personalized biomarkers be serve as an example of how to deal in future based on a Systems Biology lipidomics approach with the other in the NEJM described forms of cancer related to obesity [21].

\section{Perspectives}

Metabolomics provides a functional read-out of the physiological status of an organism [52] and when an appropriate biomarker for a complex human disease like a liposacroma (carcinogenic tumor) is found it can lead to a "Personalized medical treatment" $[53,54]$ were per individual the effects of nutritional intervention exercise protocol or drug therapy should be considered part of a lifestyle strategy to prevent or manage some types of cancer [21]. Furthermore, efforts to target the medical systems biology lipidomics based approach in oncology must consider the influence of the hypoxic tumor microenvironment and the role of cytokine release in the pathogenesis and sarcomatous change and metastasis.

\section{References}

1. Kalia M (2015) Biomarkers for personalized oncology: recent advances and future challenges. Metabolism 64: S16-21.

2. Bancroft LW, Kransdorf MJ, Peterson JJ, O'Connor MI (2006) Benign fatty tumors: Classification, clinical course, imaging appearance, and treatment. Skeletal Radiol 35: 719-733.

3. Gadgeel SM, Harlan LC, Zeruto CA, Osswald M, Schwartz AG (2009) Patterns of care in a population-based sample of soft tissue sarcoma patients in the United States. Cancer 115: 2744-2754.

4. http://apps.who.int/bookorders/anglais/detart1.jsp?codlan=1\&codcol=70\&codc $\mathrm{ch}=4005$

5. Krishnamoorthy S, Honn, KV (2011) Eicosanoids and other lipid mediators and the tumor hypoxic microenvironment. Cancer Metastasis Rev 30: 613-618.

6. Baenke F, Peck B, Miess H, Schulze A (2013) Hooked on fat: The role of lipid synthesis in cancer metabolism and tumor development. Dis Model Mech 6 : 1353-1363.

7. Signorini M, Campiglio GL (1998) Posttraumatic lipomas: Where do they really come from? Plast Reconstr Surg 101: 699-705.

8. Italiano A, Ebran N, Attias R, Chevallier A, Monticelli I, et al. (2008) NFIB rearrangement in superficial, retroperitoneal, and colonic lipomas with aberrations involving chromosome band 9p22. Genes Chromosomes Cancer 47: 971-977.

9. van Ginneken VJT, Booms R, Verheij E, de Vries E, van der Greef J (2016) The relation between non-adipose muscle fat and hepatic steatosis studied with localized $1 \mathrm{H}$ magnetic resonance spectroscopy (1H-MRS) and LCMS techniques. Anat Physiol 6:245-258.

10. van Ginneken V, Ham L, de Vries E, Verheij E, van der Greef J, et al. (2016) Comparison of hormones, lipoproteins and substrates in blood plasma in a C57BI6 mouse strain after starvation and a high fat diet: A metabolomics approach. Anat Physiol 6: 5-10.

11. Vooijs MA, Vooijs MA, Gort EH, Groot AJ, der Wall EV, van Diest PJ (2008) Hypoxic regulation of metastasis via hypoxia-inducible factors. Curr Mol Med 8: $60-67$.

12. Bertram JS (2000) The molecular biology of cancer. Mol Aspects Med 21 167-223.

13. Tavakol S (2014) Acidic $\mathrm{pH}$ derived from cancer cells may induce failed reprogramming of normal differentiated cells and turn them into cancer cells. Med Hypotheses 83: 668-672. 
Citation: Ginneken V (2017) Targeting Tumor Metabolism: A Biochemical Explanation Related to A Systems Biology Lipidomics Based Approach. J Mol Biomark Diagn S2: 022. doi:10.4172/2155-9929.S2-022

14. Wishart DS, Tzur D, Knox C, Eisner R, Guo AC et al. (2007) HMDB: The human metabolome database. Nucleic Acids Res 35: D521-526.

15. Hsu PP, Sabatini DM (2008) Cancer cell metabolism: Warburg and Beyond Cell 134: 703-707.

16. DeBerardinis RJ, Lum JJ, Hatzivassiliou G, Thompson CB (2008) The biology of cancer: Metabolic reprogramming fuels cell growth and proliferation. Cell Metab 7: 11-20.

17. van Ginneken V, Verheij E, de Vries E, van der Greef, J (2016) The discovery of two novel biomarkers for non-adipose tissue: A comprehensive LCMS study at hind limb-, heart-, carcass-muscle, liver, brain, blood plasma and food composition following a lipidomics LCMS-based approach. Cell Mol Med 2: 3.

18. Dang CV, Kim JW, Gao P, Yustein J (2008) The interplay between MYC and HIF in cancer. Nat Rev Cancer 8: 51.

19. Kroemer G, Pouyssegur J (2008) Tumor Cell Metabolism: Cancer's Achilles' Heel. Cancer Cell 13: 472-482.

20. Booth FW, Roberts CK, Laye MJ (2012) Lack of exercise is a major cause of chronic diseases. Compr Physiol 2: 1143-1211.

21. Lauby-Secretan B, Scoccianti C, Loomis D, Grosse Y, Bianchini F, et al. (2016) Body fatness and cancer-viewpoint of the IARC working group. N Engl J Med 375: 794-798.

22. Salway JG (2004) Metabolism at a Glance. Blackwell Publishing Ltd, USA.

23. Salway JG (2006) Medical Biochemistry at a glance. Blackwell Publishing Ltd, USA.

24. Trayhurn P, Alomar SY (2015) Oxygen Deprivation and the Cellular Response to Hypoxia in Adipocytes-Perspectives on white and brown adipose tissues in obesity. Front Endocrinol 6: 19

25. Netzer N, Gatterer H, Faulhaber M, Burtscher M, Pramsohler S, et al. (2015) Hypoxia, oxidative stress and fat biomolecules 5: 1143-1150.

26. van Raaij MThM (1994) Lipid metabolism in Teleost fish and the hormonal control of substrate mobilization during hypoxia and anoxia. Leiden University, Netherlands.

27. Sun RC, Denko NC (2014) Hypoxic regulation of glutamine metabolism through HIF1 and SIAH2 supports lipid synthesis that is necessary for tumor growth Cell Metab 19: 285-292.

28. van Woudenbergh GJ, Kuijsten A, van der Kallen CJ, van Greevenbroek MM, Stehouwer CD, et al. (2012) Comparison of fatty acid proportions in serum cholesteryl esters among people with different glucose tolerance status: the CoDAM study. Nutr Meta Cardiovasc Dis 22: 133-140.

29. Cook HW, McMaster CR (2002) Fatty acid desaturation and chain elongation in eukaryotes, biochemistry of lipids, lipoproteins and membranes (4thedn), Elsevier Science BV, Netherlands.

30. Santos CR, Schulze, A (2012) Lipid metabolism in cancer. FEBS J 279 2610-2623.

31. Röhrig F, Schulze A (2016) The multifaceted roles of fatty acid synthesis in cancer. Nat Rev Cancer 16: 732-749.

32. Bulla LA, Cheng TC (2013) Invertebrate models for biomedical research. Springer Science \& Business Media, USA

33. Ander BP, Dupasquier CMC, Prociuk MA, Pierce GN (2003) Polyunsaturated fatty acids and their effects on cardiovascular diseases Exp Clin Cardio 8:164-172.

34. Lopaschuk GD, Belke DD, Gamble J, Toshiyuki I, Schönekess BO (1994) Regulation of fatty acid oxidation in the mammalian heart in health and disease. Biochimica et Biophysica Acta (BBA)-Lipids and Lipid Metabolism. 1213: 263-276.

35. Hochachka PW, Lutz PL, Sick T, Rosenthal M (1993) Surviving hypoxia mechanisms of control and adaptation. CRC Press, USA.
36. Swinnen JV, Brusselmans K, Verhoeven G (2006) Increased lipogenesis in cancer cells: New players, novel targets. Curr Opin Clin Nutr Metab Care 9 : 358-365.

37. Vander Heiden MG, Lewis CC, and Craig BT (2009) Understanding the Warburg effect: The metabolic requirements of cell proliferation. Science 324 1029-1033.

38. Tran Q, Lee H, Park J, Kim SK, Park J (2016) Targeting Cancer Metabolism: Revisiting the Warburg Effects. Toxicol Res 32: 177-193.

39. Owen O, Kalhan S, Hanson R (2002) The key role of anaplerosis and cataplerosis for citric acid cycle function. J Biol Chem 277: 30409-30412.

40. Cetinbas NM, Sudderth J, Harris RC, Cebeci A, Negri GL, et al. (2016) Glucosedependent anaplerosis in cancer cells is required for cellular redox balance in the absence of glutamine. Sci Rep 6: 32606 .

41. Feron O (2009) Pyruvate into lactate and back: From the Warburg effect to symbiotic energy fuel exchange in cancer cells. Radiother Oncol 92: 329-333.

42. van Ginneken VJT (2011) Epidemiology of hunger in the world, the "HungerObesity Paradox"; its physiological and endocrinologal mechanisms, biology of starvation in humans and other organisms. Nova Science Publishers Inc, USA

43. Harisa GI, Alanazi FK (2014) Low density lipoprotein bionanoparticles: From cholesterol transport to delivery of anti-cancer drugs. Saudi Pharm J 22: 504-515.

44. Radwan A A, Alanazi, F K (2014) Targeting cancer using cholesterol conjugates Saudi Pharm J 22: 3-16.

45. van Ginneken V, Verheij E, Hekman M, Feskens E, van der Greef J, et al. (2010) The comparison of lipid profiling in Mouse brain and liver after starvation and a fatty diet: A medical systems biology approach, biology of starvation in humans and other organisms, Nova Science Publishers, USA 151-186.

46. van Ginneken V, de Vries E, Verheij E, van der Greef J (2016) Metabolomics in hind limb and heart muscle of a mouse model after a high-fat diet. Anat Physio 6: 214-223.

47. van Ginneken V, Verheij E, Poelmann R, Ramakers R, Willems-van Dijk K, et al. (2007) Metabolomics (liver and blood) profiling) in a mouse model in response to fasting: A study of hepatic steatosis. Biochimica Biophysica Acta 1771: 1263-1270.

48. Boro A, Bauer D, Born W, Fuchs B (2016) Plasma levels of miRNA-155 as a powerful diagnostic marker for dedifferentiated liposarcoma. Am J Cancer 6 : 544-552.

49. Iorio E, Ricci A, Bagnoli M, Pisanu ME, Castellano G, et al. (2010) Activation of phosphatidylcholine-cycle enzymes in human epithelial ovarian cancer cells. Cancer Res 70: 2126-2135

50. Italiano A, Di Mauro I, Rapp J, Pierron G, Auger N (2016) Clinical effect of molecular methods in sarcoma diagnosis (GENSARC): A prospective, multicenter, observational study. Lancet Oncol 17: 532-538.

51. Randall L, Cable MG (2016) The role of molecular testing in soft tissue sarcoma diagnosis. Lancet Oncol 17: 414-416.

52. Fan L, Yin M, Ke C, Ge T, Zhang G, et al. (2016) Use of plasma metabolomics to identify diagnostic biomarkers for early stage epithelial ovarian cancer. $J$ Cancer 7: 1265-1272.

53. Ramateur R, Berger R, van de Greef J, Hankemeier T (2013) Human metabolomics: Strategies to understand biology. Curr Opin Chem Bio 17 841-846.

54. van der Greef J, Hankemeier T, McBurney RN (2006) Metabolomics-based systems biology and personalized medicine: Moving towards $n=1$ clinical trials? Pharmacogenomics 7: 1087-1094.

This article was originally published in a special issue, Cancer Biomarker handled by Editor(s). Dr. Sudhir Srivastava, Cancer Biomarkers Research Group, National Institute of Health, USA; Dr. Shou-Jiang Gao, The University of Texas Health Science Centre at San Antonio, USA; Dr. Kenneth Maiese, University of Medicine \& Dentistry of New Jersey, New Jersey Medical School, USA 